\title{
PRIMEIRO REGISTRO DA ESPÉCIE INDO-PACÍFICA CHARYBDIS HELLERII (A. MILNE-EDWARDS, 1867) (CRUSTACEA: DECAPODA: PORTUNIDAE) PARA O LITORAL DO ESTADO DO CEARÁ, BRASIL
}

Recebido: $19 / 03 / 05$

Aceito: 19/04/05

\section{Luís Ernesto Arruda BEZERRA ${ }^{1^{*}}$ Alexandre Oliveira de ALMEIDA ${ }^{2}$}

\author{
${ }^{1}$ Universidade Federal do Ceará, Centro de Ciências, Departamento de \\ Biologia. Campus do Pici, Av. Mister Hull, s/n. 60455-760 Fortaleza, \\ CE, Brasil (luis_ernesto2@yahoo.com.br). \\ ${ }^{2}$ Universidade Estadual de Santa Cruz, Departamento de Ciências \\ Biológicas. Rodovia Ilhéus-Itabuna, km. 16. 45662-000 Ilhéus, BA, \\ Brasil (aalmeida@uesc.br). \\ *Autor correspondente.
}

\section{RESUMO}

Este trabalho registra pela primeira vez a ocorrência do siri originário do Indo-Pacífico Charybdis hellerii (A. Milne-Edwards, 1867) para a costa do Estado do Ceará. O material examinado foi coletado em Setembro de 2004 na praia de Redonda, município de Icapuí $\left(04^{\circ} 42^{\prime}\right.$ S; $\left.37^{\circ} 21^{\prime} \mathrm{W}\right)$, e na praia do Pontal do Maceió, município de Fortim (04 $27^{\circ}$ ' S; $37^{\circ} 04^{\prime}$ W). Os possíveis mecanismos de introdução da espécie na costa do Ceará são discutidos. Este registro amplia a distribuição setentrional conhecida para C. hellerii na costa brasileira.

Palavras-chave: Brachyura, Portunidae, Charybdis hellerii, Ceará, espécie exótica.

\begin{abstract}
First Record of the Indo-Pacific Species Charybdis Hellerii (A. Milne-Edwards, 1867) (Crustacea: Decapoda: Portunidae) From the Ceará State, Brazil

The portunid crab Charybdis hellerii (A. Milne-Edwards, 1867), a species of Indo-Pacific origin, is reported for the first time from the coast of Ceará State, Brazil. The material examined was collected in

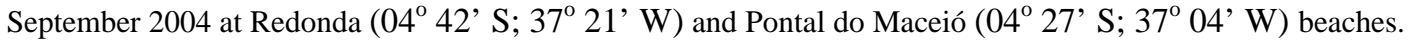
We discuss the possible ways of introduction of this species on Ceará coast. The present record extends the geographic range known for this species in the Brazilian coast.
\end{abstract}

Key words: Brachyura, Portunidae, Charybdis hellerii, Ceará State, nonindigenous species, invasive species.

\section{INTRODUÇÃO}

Os crustáceos decápodos são, de maneira geral, particularmente bem adaptados para migrações de longa distância e ocupação de novas localidades (RODRÍGUEZ; SUÁREZ, 2001).

Charybdis hellerii é uma espécie de siri originária do Indo-Pacífico que foi introduzida no Mar Mediterrâneo após a abertura do Canal de Suez, no final do século XIX (GALIL, 1992; 
RODRÍGUEZ; SUÁREZ, op cit.). No Atlântico Ocidental a espécie foi coletada pela primeira vez em Cuba, no fim dos anos 80 (GÓMEZ; MARTÍNEZ-IGLESIAS, 1990), sendo subseqüentemente coletada na Venezuela (BOLAÑOS; HERNÁNDEZ; HERNÁNDEZ; MARTÍNEZ-IGLESIAS, 1997) e na costa caribenha da Colômbia (CAMPOS; TÜRKAY, 1989). Posteriormente, Lemaitre (1995) registrou a ocorrência de C. hellerii para a costa da Flórida.

A introdução dessa espécie nas Américas provavelmente deu-se através de transporte de formas larvais em "água de lastro" de navios oriundos do Mediterrâneo Oriental. Gómez; MartínezIglesias (op cit.) e Campos; Türkay (op cit.), afirmam que durante a década de 80 houve um incremento notável do fluxo de navios entre o Mediterrâneo e o Caribe. Já a ocorrência desse portunídeo em outras regiões caribenhas e na Flórida poderia ser explicada pela dispersão de larvas através das correntes oceânicas (LEMAITRE, 1985). Atualmente o caso de C. hellerii é tido como exemplo de introdução bem sucedida de uma espécie exótica marinha no Atlântico (TAVARES; AMOUROUX, 2003). Sua fase larval (cerca de 44 dias em condições de laboratório), é duas vezes mais longa do que as três semanas gastas por um navio no percurso entre o Mediterrâneo e águas costeiras do Atlântico Ocidental (DINEEN et al., 2001).

As primeiras publicações sobre a ocorrência dessa espécie na costa do Brasil datam do ano de 1996 (CALADO, 1996; CARQUEIJA; GOUVÊA, 1996; NEGREIROS-FRANSOZO, 1996; TAVARES; MENDONÇA JR, 1996), tendo sido posteriormente registrada em diversas localidades costeiras brasileiras. Dada a grande extensão do litoral do país, é possível que a introdução do portunídeo tenha se dado em mais de um ponto, tendo posteriormente se dispersado na forma larval via correntes para várias outras áreas costeiras (MANTELATTO; DIAS, 1999). Este trabalho registra pela primeira vez a ocorrência de C. hellerii para o litoral do Estado do Ceará.

\section{MATERIAL E MÉTODOS}

Os espécimes descritos neste trabalho foram coletados durante as atividades do projeto “Zoneamento Ecológico e Econômico do Estado do Ceará (ZEE da Zona Costeira)”, promovido pelo Instituto de Ciências do Mar (LABOMAR) em convênio com a Superintendência Estadual do Meio Ambiente (SEMACE). Os exemplares foram capturados manualmente na região entre-marés, em substrato consolidado, nas praias de Redonda (município de Icapuí, Ceará) e Pontal do Maceió (município de Fortim, Ceará), ambas localizadas à leste da cidade de Fortaleza. Após a coleta, os exemplares foram fixados em etanol 70\% e identificados. Os animais serão depositados na Coleção Carcinológica do Instituto de Ciências do Mar (LABOMAR) da Universidade Federal do Ceará, Fortaleza. No item material examinado são utilizadas as seguintes abreviaturas: $\mathrm{CC}=$ comprimento da carapaça e LC = largura da carapaça.

\section{RESULTADOS}

Família Portunidae Rafinesque, 1815

Charybdis hellerii (A. Milne-Edwards, 1867)

Material examinado: 1 macho (CC X LC = 27,5 X 43,4 mm) e 1 fêmea (CC X LC = 27,7 X 41,5 mm), 26.IX.2004, Praia de Redonda, Icapuí, Ceará ( $04^{\circ} 27^{\prime}$ S; $37^{\circ} 04^{\prime}$ W), L. E. A. Bezerra col.; 1 macho (CC X LC = 21,4 X 32,4 mm) e 1 fêmea (CC X LC = 24 X 38,1 mm), 27.IX.2004, Praia do Pontal do Maceió, Fortim, Ceará (04 ${ }^{\circ} 27^{\prime}$ S; $37^{\circ} 04^{\prime}$ W), L. E. A. Bezerra col..

Distribuição geográfica: Mar Vermelho, Djibouti, Somália, África do Sul, Madagascar, Golfo Pérsico, Hong Kong, Singapura, Ceilão, Índia, China, Japão, Indonésia, Filipinas, Nova Caledônia,

Tropical Oceanography, Recife, v. 33, n. 1, p. 33-38, 2005. 
Austrália e Havaí. Mediterrâneo Oriental: Egito e Israel. Atlântico Ocidental: Flórida, Cuba, Colômbia, Venezuela, Guiana Francesa e Brasil (CALADO, 1996; DINEEN et al., 2001; TAVARES; AMOUROUX, 2003).

Registros anteriores para o Brasil: Rio Grande do Norte: Ferreira; Sankarankutty; Cunha; Duarte (2001), Ferreira; Sankarankutty (2002). Pernambuco: Coelho; Santos (2003). Alagoas: Calado (op cit.). Bahia: Carqueija; Gouvêa (1996), Almeida; Coelho; Santos (2003). Rio de Janeiro: Tavares; Mendonça Jr (1996). São Paulo: Mantelatto; Correa (1996), Negreiros-Fransozo (1996), Mantelatto; Souza-Carey (1998), Mantelatto; Dias (1999), Mantelatto; Fransozo (2000), Mantellato; Garcia (2001), Mantelatto; O’Brien; Biagi (2003), Bertini; Fransozo; Melo (2004). Santa Catarina: Mantelatto; Dias (op cit.).

Habitat: Preferência por sedimentos não consolidados, podendo, entretanto, ser encontrada em sedimentos consolidados, como rochas e corais. Juvenis podem ser encontrados associados a briozoários como Schizoporella unicornis (MANTELATTO; SOUZA-CAREY, op cit.). Há ainda registros de espécimes associados à raízes de árvores de manguezal (Rhizophora mangle) (CAMPOS; TÜRKAY, 1989; DINEEN et al., op cit.). Do entre-marés até 51m de profundidade.

Identificação: Características diagnósticas da espécie podem ser obtidas em Lemaitre (1995). Uma chave para identificação dos gêneros da família Portunidae do Brasil, que inclui o gênero Charybdis, pode ser encontrada em Tavares; Mendonça Jr (1996).

\section{DISCUSSÃO}

O transporte de espécies através da "água de lastro" de navios é um dos mecanismos mais efetivos para a introdução de espécies exóticas de decápodos (CARLTON, 1985), onde as espécies invasoras podem afetar de várias maneiras as nativas, através da competição por alimento ou abrigo e/ou predação sobre outros membros da comunidade. Indiretamente, os organismos invasores podem afetar as espécies nativas pela introdução de doenças (RODRÍGUEZ; SUÁREZ, 2001).

Próximo à região de coleta dos espécimes, localiza-se o porto-ilha de Areia Branca, situado há cerca de $14 \mathrm{~km}$ da costa do Rio Grande do Norte $\left(04^{\circ} 49^{\prime} \mathrm{S} ; 37^{\circ} 02^{\prime} \mathrm{W}\right)$, cuja finalidade é escoar a produção das salinas do referido Estado. Esse porto pode ter sido o possível local de introdução de C. hellerii na costa do Ceará, visto que as correntes marinhas favorecem o transporte de larvas do porto para o litoral desse Estado. Uma possibilidade que não pode ser descartada é que o transporte de indivíduos tenha se dado através do casco de embarcações, visto que alguns tipos possuem compartimentos nos quais ocorre captura de água com a finalidade de resfriar o motor, abastecimento dos tanques de lastro, lavagem do convés e combate a eventuais incêndios (COELHO; SANTOS, 2003).

Na costa do Ceará existem diversos ecossistemas favoráveis ao estabelecimento e proliferação de $C$. hellerii. Assim, o portunídeo é potencialmente capaz de competir por alimento e espaço com as espécies nativas do gênero Callinectes, conforme sugerido por Dineen (2001).

O presente registro de $C$. hellerii para o Ceará amplia a distribuição geográfica conhecida para essa espécie na costa brasileira, sendo este o único registro existente entre o Estado do Rio Grande do Norte (FERREIRA; SANKARANKUTTY; CUNHA; DUARTE, 2001), e a Guiana Francesa (TAVARES; AMOUROUX, 2003). Resta ainda uma ampla lacuna na distribuição desse portunídeo no Norte brasileiro que pode possivelmente estar relacionada com a falta de trabalhos recentes nesta porção do litoral do país. Faz-se necessária a realização de estudos sobre a distribuição, biologia e ecologia da espécie na região de modo a avaliar os possíveis impactos de sua introdução sobre a comunidade nativa.

Tropical Oceanography, Recife, v. 33, n. 1, p. 33-38, 2005. 


\section{AGRADECIMENTOS}

Os autores agradecem ao Dr. Petrônio Alves Coelho, do Departamento de Oceanografia (UFPE) pelo encorajamento da publicação. L. E. A. Bezerra agradece à Profa. Dra. Helena Matthews-Cascon do Departamento de Biologia da UFC e ao Biólogo do Instituto de Ciências do Mar da UFC, M. Sc. Wilson Franklin Júnior, pela ajuda na coleta do material. A. O. de Almeida agradece à FAPESB (Fundação de Amparo à Pesquisa do Estado da Bahia) pela concessão de uma bolsa de produtividade em pesquisa no período no qual foi realizado este trabalho.

\section{REFERÊNCIAS BIBLIOGRÁFICAS}

ALMEIDA, A. O.; COELHO, P. A.; SANTOS, J. T. A. New records of decapod crustaceans (Dendrobranchiata and Brachyura) for the state of Bahia, Brazil. Nauplius, v. 11, n. 2, p. 129-133, 2003.

BERTINI, G.; FRANSOZO, A; MELO, G. A. S. Biodiversity of brachyuran crabs (Crustacea: Decapoda) from non-consolidated sublittoral bottom in the northern coast of São Paulo state, Brazil. Biodiversity and Conservation, v. 13, p. 2185-2207, 2004.

BOLAÑOS, J. A.; HERNÁNDEZ, G.; HERNÁNDEZ, J. E.; MARTÍNEZ-IGLESIAS, J. C. Presencia de Charybdis hellerii (A. Milne-Edwards, 1867) (Crustacea: Decapoda: Portunidae) en aguas marinas de Venezuela. Avicennia, v. 6/7, p. 147-148, 1997.

CALADO, T. C. S. Registro de Charybdis hellerii (Milne Edwards, 1867) em águas do litoral brasileiro (Decapoda: Portunidae). Boletim de Estudos de Ciências do Mar, v. 9, p. 175-180, 1996.

CAMPOS, N. H.; TÜRKAY, M. On a record of Charybdis hellerii from the Caribbean coast of Colombia (Crustacea: Decapoda: Portunidae). Senckenbergiana Maritima, v. 20, n. 3/4, p. 119123, 1989.

CARLTON, J. T. Transoceanic and interoceanic dispersal of coastal marine organisms: The biology of ballast water. Oceanography and Marine Biological Annual Review, v. 23, p. 313-371, 1985.

CARQUEIJA, C. R. G.; GOUVÊA, E. P. A ocorrência, na costa brasileira, de um Portunidae (Crustacea, Decapoda), originário do Indo-Pacífico e Mediterrâneo. Nauplius, v. 4, p. 105-112, 1996.

COELHO, P. A.; SANTOS, M. C. F. Ocorrência de Charybdis hellerii (Milne Edwards, 1867) (Crustacea, Decapoda, Portunidae) no litoral de Pernambuco. Boletim Técnico-Científico do CEPENE, v. 11, p. 167-173, 2003.

DINEEN, J. F. Charybdis hellerii. Fort Pierce: Smithsonian Marine Station at Fort Pierce, 2001. Disponível em. Acesso em 22 de mar. 2005.

DINEEN, J. F.; CLARK, P. F.; HINES, A. H.; REED, S. A.; WALTON, H. P. Life history, larval description, and natural history of Charybdis hellerii (Decapoda, Brachyura, Portunidae), an 
invasive crab in the western Atlantic. Journal of Crustacean Biology, v. 21, n. 3, p. 774-805, 2001.

FERREIRA, A. C.; SANKARANKUTTY, C. Estuarine carcinofauna (Decapoda) of Rio Grande do Norte, Brazil. Nauplius, v. 10, n. 2, p. 121-129, 2002.

FERREIRA, A. C.; SANKARANKUTTY, C.; CUNHA, I. M. C.; DUARTE, F. T. Yet another record of Charybdis helleri (A. Milne Edwards) (Crustacea, Decapoda) from the northeast of Brazil. Revista Brasileira de Zoologia, v. 18 (Supl. 1), p. 357-358, 2001.

GALIL, B. Eritrean decapods in the Levant. Biogeography in motion. Bulletin de l'Institute Océanographique, Monaco, ${ }^{\circ}$ especial 9, p. 115-123, 1992.

GÓMEZ, O.; MARTÍNEZ-IGLESIAS, J. C. Reciente hallazgo de la especie indopacífica Charybdis helleri (A. Milne-Edwards, 1867) (Crustacea: Decapoda: Portunidae) en aguas cubanas. Caribbean Journal of Science, v. 26, n. 1/2, p. 70-72, 1990.

LEMAITRE, R. Charybdis helleri (Milne Edwards, 1867), a nonindigenous portunid crab (Crustacea: Decapoda: Brachyura) discovered in the Indian River lagoon system of Florida. Proceedings of the Biological Society of Washington, v. 108, n. 4., p. 643-648, 1995.

MANTELATTO, F. L. M.; CORREA, E. K. Composition and seasonal variation of the brachyuran crabs (Crustacea, Decapoda) living on Sargassum cymosum in the Ubatuba region, São Paulo, Brazil. Bioikos, v. 9/10, n. 1/2, p. 22-31, 1996.

MANTELATTO, F. L. M.; DIAS, L. L. Extension of the known distribution of Charybdis hellerii (A. Milne Edwards, 1867) (Decapoda, Portunidae) along the western tropical South Atlantic. Crustaceana, v. 72, n. 6, p. 617-620, 1999.

MANTELATTO, F. L. M.; FRANSOZO, A. Brachyuran community in Ubatuba bay, northern coast of São Paulo state, Brazil. Journal of Shellfish Research, v. 19, n. 2, p. 701-709, 2000.

MANTELATTO, F. L. M.; GARCIA, R. B. Biological aspects of the nonindigenous portunid crab Charybdis hellerii in the western tropical South Atlantic. Bulletin of Marine Science, v. 68, n. 3, p. 469-477, 2001.

MANTELATTO, F. L. M.; SOUZA-CAREY, M. M. Brachyura (Crustacea, Decapoda) associated to Schizoporella unicornis (Bryozoa, Gymnolaemata) in Ubatuba Bay (SP), Brazil. Brazilian

Archives of Biology and Technology, v. 41, n. 2, p. 212-217, 1998.

MANTELATTO, F. L. M.; O’BRIEN, J.; BIAGI, R. Parasites and symbionts of crabs from Ubatuba bay, São Paulo State, Brazil. Comparative Parasitology, v. 70, n. 2, p. 211-214, 2003.

NEGREIROS-FRANSOZO, M. L. The zoea I of Charybdis hellerii (A. Milne-Edwards, 1867) (Decapoda, Portunidae) obtained in laboratory. Nauplius, v. 4, p. 165-168, 1996.

RODRÍGUES, G.; SUÁREZ, H. Anthropogenic dispersal of decapod crustaceans in aquatic environments. Interciencia, v. 26, n. 7, p. 282-288, 2001. 
TAVARES, M.; AMOUROUX, J. M. First record of the non-indigenous crab, Charybdis hellerii (A. Milne-Edwards, 1867) from French Guyana (Decapoda, Brachyura, Portunidae). Crustaceana, v. 76, n. 5, p. 625-630, 2003.

TAVARES, M.; MENDONÇA JR, J. B. Charybdis hellerii (A. Milne Edwards, 1867) (Brachyura: Portunidae), eight nonindigenous marine decapod recorded from Brazil. Crustacean Research, v. 25, p. 151-157, 1996.

Tropical Oceanography, Recife, v. 33, n. 1, p. 33-38, 2005. 